A Journal of Agricultural Science Published by the California Agricultural Experiment Station

\title{
RATE OF PIGMENT \\ DEGRADATION IN THE PHLOEM OF DEHYDRATED RED CORE CHANTENAY CARROTS
}

\author{
ELLIOT WEIER
}




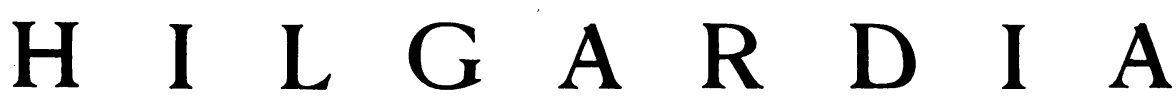

A Journal of Agricultural Science Published by the California Agricultural Experiment Station

\section{RATE OF PIGMENT DEGRADATION IN THE PHLOEM OF DEHYDRATED RED CORE CHANTENAY CARROTS ${ }^{1}$}

\author{
ELLIOT WEIER ${ }^{2}$
}

\section{INTRODUCTION}

The DETERIORATION of dehydrated carrots in storage involves complex reactions. Although detailed information about these changes is not available at present, the following general facts are known: (1) the pigments present disappear; (2) haylike and other off-odors develop; (3) changes in the oil droplets within the cells result in a positive test for aldehydes with Schiff's reagent; (4) the carrots darken (apparently because the amino acids combine with sugars), and the substance responsible for this change in color is watersoluble; (5) blanching inactivates enzymes and also modifies other changes that occur in storage; (6) sulphite preserves color, especially by preventing the darkening reaction and by acting somewhat as an antioxidant; (7) other changes unquestionably occur.

In preliminary studies on carrots during processing and storage, the overall changes in pigment concentration were expected to serve as a marker for at least some of the oxidations taking place in the lipid phase. This assumption was based on the following facts : (1) concentration of pigment may change greatly in storage; (2) carotene is a pro-oxidant (Olcott and Mattill, 1936a) ${ }^{3}$ and its breakdown in oil solution results from the previous oxidation of the oil (Strain, 1941; and Sumner, 1942) ; (3) carotene in blanched and in dried carrots is dissolved in oil droplets (Weier, 1944a).

This paper reports experiments to test the stability of the pigment complex in carrots after various treatments. These treatments include blanching, leaching, and soaking in buffers, in solutions of high and low $\mathrm{pH}$, in organic and mineral acids, and in antioxidants. Pigment concentration has been measured on the Evelyn colorimeter. Thus far, no attempt has been made to isolate individual pigments or to follow the details of their breakdown.

\footnotetext{
${ }^{1}$ Received for publication September 18, 1945.

${ }^{2}$ Associate Professor of Botany and Associate Botanist in the Experiment Station.

${ }^{3}$ See "Literature Cited" for citations, which are referred to in the text by author and date.
} 


\section{METHODS}

Phloem was secured from five plantings of Red Core Chantenay carrots. The carrots, which were grown at Davis, were harvested immediately before use. Seed was sown in April and September, 1943 (plantings 1 and 2) ; in April and September, 1944 (plantings 3 and 4); and in April, 1945 (planting 5).

The technique used was the same as that previously reported by Weier $(1944 b)$, except that more uniform samples were obtained by a careful distribution of carrot cylinders. That this greater uniformity produced good agreement between duplicate samples of the same experiment, and between similar experiments, is shown by figure 1 . Curves 1 to 4 (fig. 1 ) are plots of carotene degradation in blanched carrot cylinders held at $60^{\circ} \mathrm{C}$ in moist air for the time intervals indicated. Curves 1 and 2 are taken from experiments run on October 16 and 18. Curve 4 is from an experiment of June 27. Curve 3 is the average of ten experiments on pigment breakdown in blanched, summerand fall-harvested carrots. The greatest difference obtained in these experiments is shown by curves 1 and 4 . Curve 5 represents the carotene degradation in unblanched carrot cylinders. The points obtained in this experiment are given on the graph; agreement was equally good in other curves.

For the experiments with dried carrots two types were used: (1) Carrots, cut into cubes approximately $1 \mathrm{~cm}$ on edge, were blanched, treated, and dried for 20 hours in a laboratory drying oven. They were then stored in glass jars at $40^{\circ} \mathrm{C}$ for 3 months, and the carotene was determined at 30-day intervals. The moisture content was about 7 per cent. (2) Carrots were blanched, treated, and dried in an experimental dehydrater made by members of the Agricultural Engineering Division. The drying time was 5 hours.

After drying, both types were ground to pass a 20 -mesh screen. All powder small enough to pass a 48-mesh screen was sifted out. The moisture content averaged 5 per cent. For pigment determinations, boiling water was poured over a weighed sample of the powder and it was then allowed to stand for 1 hour. Water-soluble pigments that developed during storage were washed out. The carotinoid pigment was extracted in acetone. Determinations were made with an acetone extract in the Evelyn colorimeter. The usual precautions for protecting carotene solutions against light were observed. Most colorimetric examinations were made within 2 hours after extraction, and when this was not possible, solutions were held at $4^{\circ} \mathrm{C}$ until examined. All determinations were completed within 18 hours after extraction. In this 18-hour interval, no change occurred in the pigment held at $4^{\circ}$.

Dilutions of the pigment extracts showed a straight-line relation when examined on the Evelyn colorimeter, provided the galvanometer readings were kept between 30 and 70 . The deviation from a straight line beyond 70 was considerable. Results were calculated in terms of the optical density of pigment per gram of fresh carrots. Since constants have been worked out with Eastman Kodak carotene (10 per cent A, 90 per cent B), the results could be expressed in micrograms of this sample. Such a procedure, however, seemed unwise, because neither the pigments of the carrot extract nor the detailed changes occurring in these pigments have been analyzed. Unquestionably, 
however, the density as measured by the Evelyn colorimeter represents concentration of pigments. To facilitate comparisons between experiments, results have been expressed on a percentage basis.

\section{RESULTS}

Age and Season. Carotene content varies with age and season. Planting 3 was sampled over a period of 10 months; other plantings for shorter periods. The first carrots selected for experimentation in planting 3 were 100 days old. Expressed in terms of Eastman carotene, the phloem in these carrots contained about 88 micrograms of carotene per gram of fresh carrot. During the next 30 days, the amount of pigment doubled to 176 micrograms per gram of

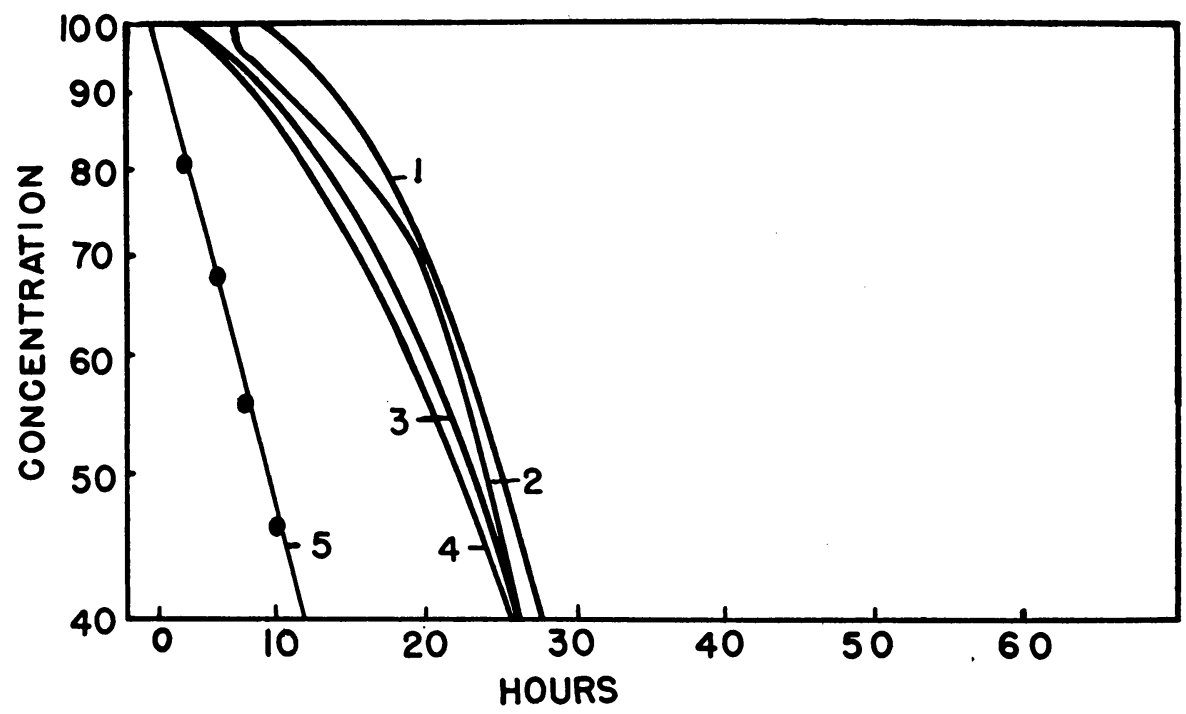

Fig.1. Rate of carotene breakdown in the phloem of summer-and fall-harvested carrots: curves 1,2 , and 4 , separate experiments on blanched phloem; curve 3 , average of 10 summer and fall experiments on blanched phloem; curve 5, summer experiment on unblanched phloem.

fresh carrot phloem. This concentration remanied fairly constant from the middle of July to the middle of October. The amount then increased rather rapidly to 264 micrograms, which remained constant for the remainder of the experiment. Higher figures were occasionally obtained, the highest being 320 micrograms in a late-October experiment. Judging from the evidence, the pigment of California-grown carrots increases slowly for as long a time as 7 months, and does not remain constant after 130 days, as Barnes (1936) reports for carrots grown in New York. There is no evidence that the amount of carotene decreases during the California rainy season.

Unblanched Carrots. Pigment concentration of fresh carrots plotted against time on semilog paper results in a straight line (fig. 1, curve 5) and therefore follows a first-order reaction. Zero time was determined by extrapolation of the curve to 100 per cent carotene. The $k$ value for the curve shown

\footnotetext{
In calculating $k$ from the expression $\left(\frac{1}{t} \log \frac{a}{2-x}\right)$, the time is in hours, the logarithm
to base $e$.
} 
was 10.7 at 4 hours; it remained practically constant over 30 hours, varying only from 9.05 to 9.88 .

The rate of pigment breakdown in unblanched carrots varied with the season. This variation is shown in table 1 . The pigment was least stable in the fall, as is indicated by a high value for $k$. Its stability increased during the winter, and as the carrots aged. The rate of pigment degradation was most rapid by the end of 4 hours' incubation in all experiments except one-that carried out on April 9, when no breakdown had occurred at 4 hours.

In sample 4 (planted in September, 1944) the rate of pigment disappearance agreed closely with that noted in the older sample when the roots of both

TABLE 1

Rate of Carotene Breakdown, as Indicated by the Specific Rate Constant $k$, in Unblanched Carrots Harvested at Different Seasons and InCUBATEd at $60^{\circ} \mathrm{C}$ in MoIst Air

\begin{tabular}{|c|c|c|c|c|c|c|c|c|c|c|c|}
\hline \multirow{2}{*}{ Hours } & \multicolumn{2}{|c|}{ Planted 1943} & \multicolumn{6}{|c|}{ Planting 3, planted April, 1944} & \multicolumn{3}{|c|}{$\begin{array}{l}\text { Planting 4, planted } \\
\text { September, } 1944\end{array}$} \\
\hline & $\begin{array}{c}\text { Feb. } \\
9 \\
1944\end{array}$ & $\begin{array}{c}\text { Feb. } \\
10 \\
1944\end{array}$ & $\begin{array}{c}\text { Sept. } \\
15, \\
1944\end{array}$ & $\begin{array}{c}\text { Oct. } \\
23, \\
1944\end{array}$ & $\begin{array}{c}\text { Nov. } \\
14, \\
1944\end{array}$ & $\begin{array}{c}\text { Dec. } \\
15 \\
1944\end{array}$ & $\begin{array}{c}\text { Feb. } \\
2, \\
1945\end{array}$ & $\begin{array}{c}\text { April } \\
9 \\
1945\end{array}$ & $\begin{array}{c}\text { March } \\
15, \\
1945\end{array}$ & $\begin{array}{c}\text { March } \\
22, \\
1945\end{array}$ & $\begin{array}{c}\text { April } \\
23, \\
1945\end{array}$ \\
\hline & $k$ & $k$ & $k$ & $k$ & $k$ & $k$ & $k$ & $k$ & $k$ & $k$ & $k$ \\
\hline 4 & $\ldots \ldots$ & $\cdots \cdots$ & $\ldots$ & 10.6 & .. & $\ldots$ & 3.8 & 0 & $\ldots$ & $\ldots$ & $\ldots$ \\
\hline 6 & & $\ldots$ & 3.2 & 9.7 & 6.7 & 5.6 & $\ldots$ & $\ldots$ & $\ldots$ & $\ldots$ & $\ldots$ \\
\hline 8 & $\ldots \ldots$ & $\ldots \ldots$ & 3.1 & 9.9 & 6.5 & 4.8 & 5.8 & 2.1 & 2.4 & 7.4 & 6.4 \\
\hline 21 & 4.4 & 4.0 & 3.1 & 9.7 & 4.7 & 4.7 & 3.9 & 1.9 & 1.7 & 6.5 & 5.7 \\
\hline 26 & $\ldots \ldots$ & $\ldots \ldots$ & 4.7 & 9.5 & 4.6 & 4.6 & 3.1 & 2.0 & 1.4 & 5.4 & 10.0 \\
\hline
\end{tabular}

samples were dormant (March 15). When spring growth was resumed (March 22 ), however, the pigment was much less stable in the younger roots than in the older ones. The spring season was such that both plantings bolted upon the resumption of growth. The carrots sown in September of the previous year (planting 2) had grown vegetatively several months before bolting. Although rate studies were not carried out on this planting, the figures available indicate a rapid breakdown of the pigment in spring-harvested carrots.

A series of four experiments run during February, 1944, in which unblanched carrots were treated like those studied in February, 1945, gave the following values for $k$ after 21 hours' incubation : 3.95, 4.37, 4.23, and 3.95.

Blanched Carrots. Season also affected the stability of carotene in blanched undried carrots. During a given season, however, the stability of the carotene was constant. Good agreement was obtained between experiments on a single culture carried out during the summer and fall of 1944 (fig. 1, curves 1 to 4 ). In winter and spring the pigment in the roots of this same planting was more stable (fig. 2 , planting 3 , winter). The agreement among nine experiments was good; the curve shown in figure 2 is the average. Furthermore, different plantings harvested in the same season of different years agreed well in rate of carotene breakdown (fig. 2). There is one exception-compare spring-harvested carrots of plantings 2 and 4 . These carrots were planted in September of 1943 and 1944, respectively, and harvested in the springs of 1944 and 1945. Planting 2, however, had a long growing season in the spring 
of 1944, whereas planting 4 bolted immediately upon the resumption of growth. Pigment broke down rapidly in the growing, spring-harvested carrots of planting 2, but remained stable in the bolting, spring-harvested carrots of planting 4. Planting 5 was sown in April, 1945. When first examined in July, the roots were growing vigorously. The results (fig. 2, summer, 5) agree closely with those obtained for other summer-harvested roots.

In eighteen out of twenty experiments on blanched carrots, the rate of pigment breakdown was slow at the start of the experiment, but increased at longer incubation periods. The two exceptions were obtained with winter-

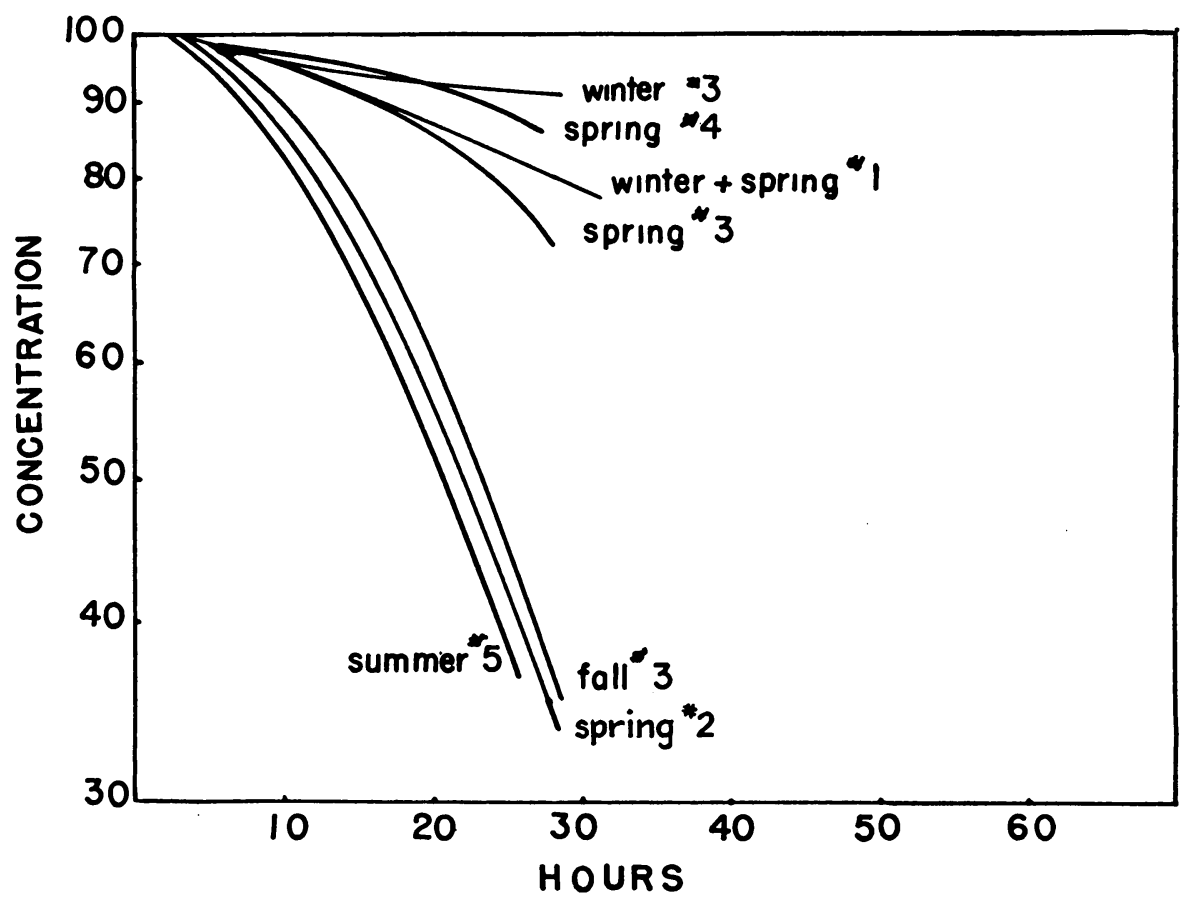

Fig. 2. Rate of carotene breakdown in blanched cylinders of carrot phloem. Seasons are indicated. Numbers refer to planting of carrots.

harvested roots. Values for $k$, calculated as the constant for a first-order reaction during the summer, ranged from 0.00 at 4 and 8 hours to 3.80 at 26 hours. In winter the values of $k$ ranged from 0.00 at 4 and 8 hours to approximately 0.48 at 26 hours.

Judging from the course of the reaction as shown by these results, the summer-harvested carrots contained an inhibitor, which slowed up the reaction during the first 8 hours of incubation. As the inhibitor was destroyed, the rate of pigment degradation increased.

Leaching Blanched Undried Carrots. It was previously reported (Weier, $1944 b$ ) that the stability of carotene is lowered if the carrot dice are washed after blanching, and that this is more apparent in winter than in summer. These results have been corroborated and extended (fig. 3). Curves 1 and 1 leach (fig. 3) show the difference in rate of carotene breakdown in blanched 
dice (summer-harvested) when washed after blanching (curve 1 leach) and when not so washed (curve 1). Similarly, curves 2 and 2 leach show the rate of pigment breakdown for winter-harvested carrots. Carrots were washed in running water for 20 minutes immediately after blanching. The difference between washed and unwashed dice was several times greater in winter-harvested than in summer-harvested carrots. Carotene was more stable in winterharvested carrots, however, even after washing, than in unwashed summer carrots.

Leaching Blanched Dehydrated Carrots. Washing after blanching lessened the stability of the pigment in dried blanched carrots - a fact illustrated

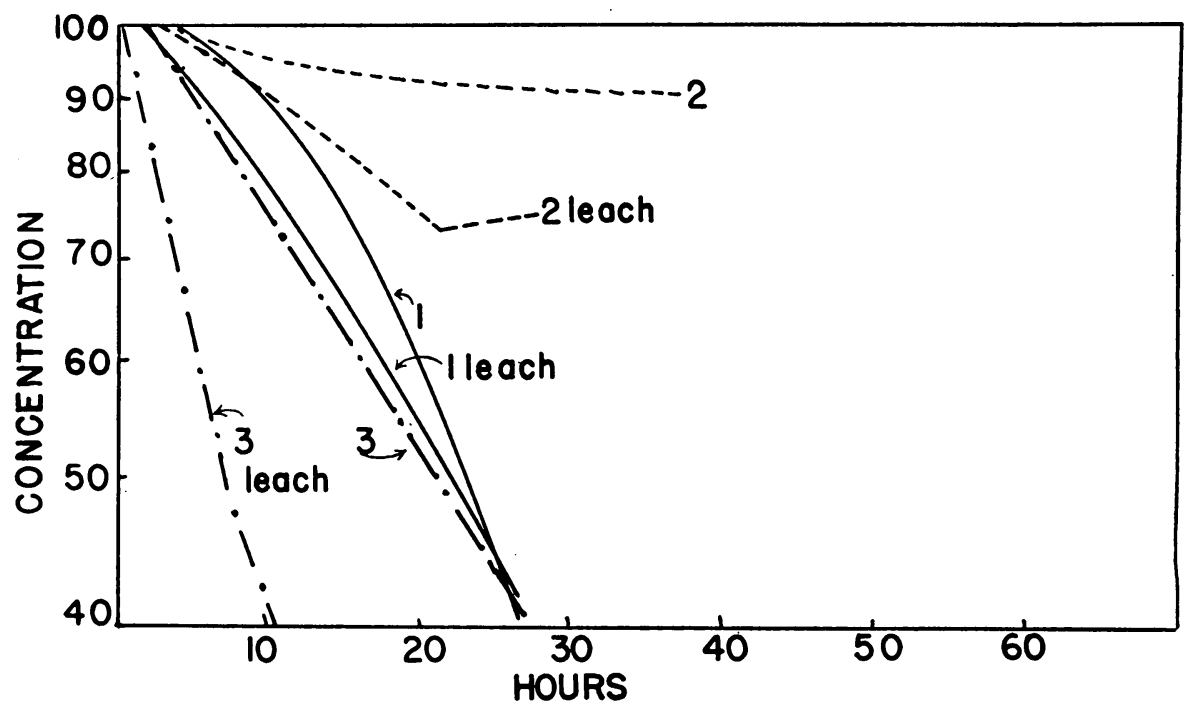

Fig. 3. Rate of carotene breakdown in carrot phloem washed after blanching, as contrasted with unwashed blanched carrot phloem. Curves 1 and 1 leach, summer-harvested carrots, 1944. Curves 2 and 2 leach, winter-harvested carrots, 1944, 1945. Curves 3 and 3 leach, spring-harvested dried carrots, ground, stored in oxygen at $60^{\circ} \mathrm{C}$, and analyzed at the hours indicated.

by the curves in figure 4 . In this series of experiments, carrots were diced and blanched. After one half of the dice had been soaked in water for 20 minutes, both samples were dried and stored at $40^{\circ} \mathrm{C}$ for 3 months. Analyses were made every 30 days.

In seven out of eleven experiments on blanched washed carrots carried out between July 7 and October 31, the specific rate constant varied but slightly during the course of the experiment. It varied, for example, from 2.34 to 2.72 ; from 2.02 to 2.36 ; and from 2.48 to 2.52. In the other four experiments it increased during the course of the experiment-for example, from 1.04 to 2.42 and from 1.41 to 2.74. Although this indicates some retardation in the rate of breakdown during the initial hours of the experiment,the retardation is less than in the unwashed blanched carrots. (Compare 1.04 to 2.42 for blanched washed carrots with 0.00 to 3.80 for blanched unwashed carrots.) In these latter four experiments it may be assumed that the inhibitor was not completely removed by the leaching. 


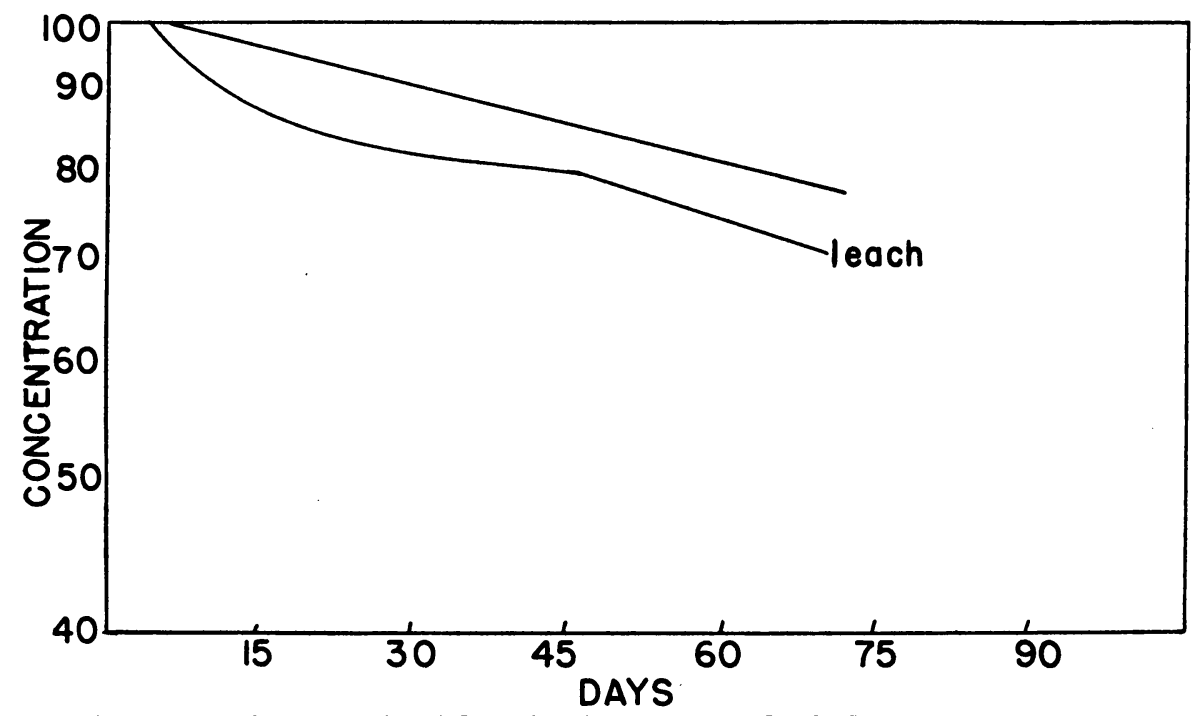

Fig. 4. Rate of carotene breakdown in winter-harvested dehydrated carrots stored at $40^{\circ} \mathrm{C}$ and analyzed monthly.

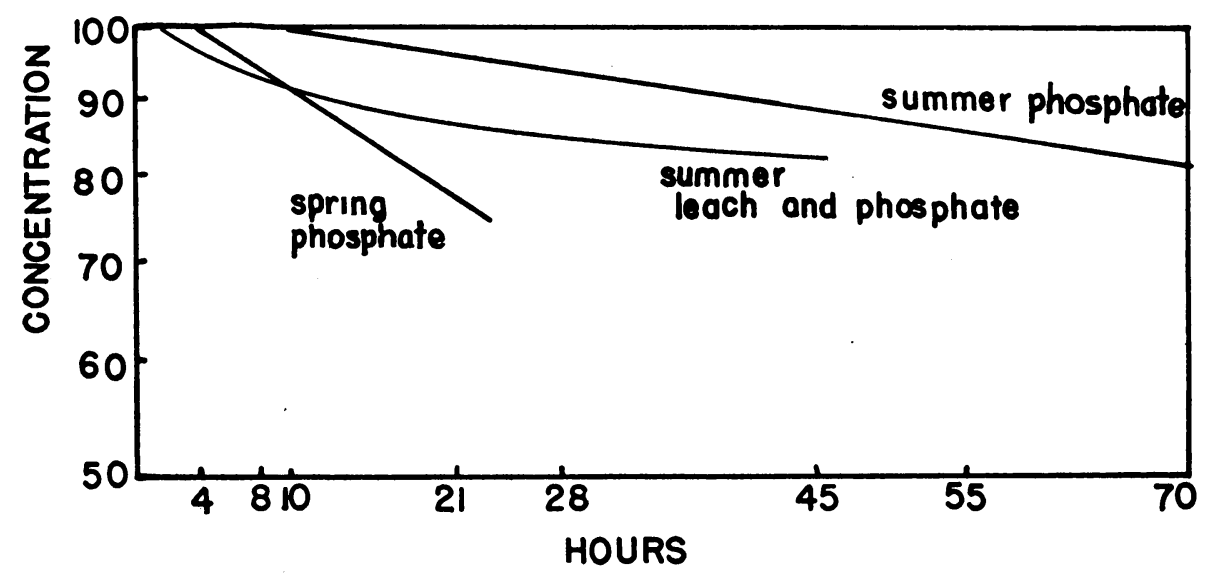

Fig. 5. Rate of carotene breakdown in cylinders of summer-harvested, blanched carrot phloem that was impregnated with standard phosphate buffer at $\mathrm{pH}$ 7.1.

In still another series of experiments, carrots were blanched; one half of the sample was leached in distilled water. They were dried and ground. These samples were incubated over oxygen at $60^{\circ} \mathrm{C}$. The results are shown in curves 3 and 3 leach (fig. 3 ). The carotene in the leached sample broke down more rapidly than that in the blanched, unleached sample.

The evidence obtained indicates that when blanched carrots are washed, some substance that acts to retard the breakdown of pigment is removed.

Buffer Treatments-Undried Carrots. Pigments in summer-harvested carrots that had been blanched and treated with phosphate buffers close to neutrality, were stable when incubated at $60^{\circ} \mathrm{C}$ in moist air (fig. 5, summer). The pigments in summer-harvested carrots that were leached after blanching 
and then treated with neutral phosphate buffer were stable (fig. 5, summer, leach and phosphate). In these experiments the blanched carrots were soaked in the buffer for 20 minutes and were kept moistened with it during incubation. In the tests that furnished the data for the spring curve of figure 5, the blanched dice were soaked for 20 minutes in the buffer. During incubation the dice were moistened with distilled water ; from these, soluble phosphorus compounds diffused during incubation (fig. 7). The results indicate that the breakdown of pigment was retarded somewhat by soaking the dice in neutral phosphate buffers before incubation. (Compare fig. 5 with fig. 2 , summer and fall curves.)

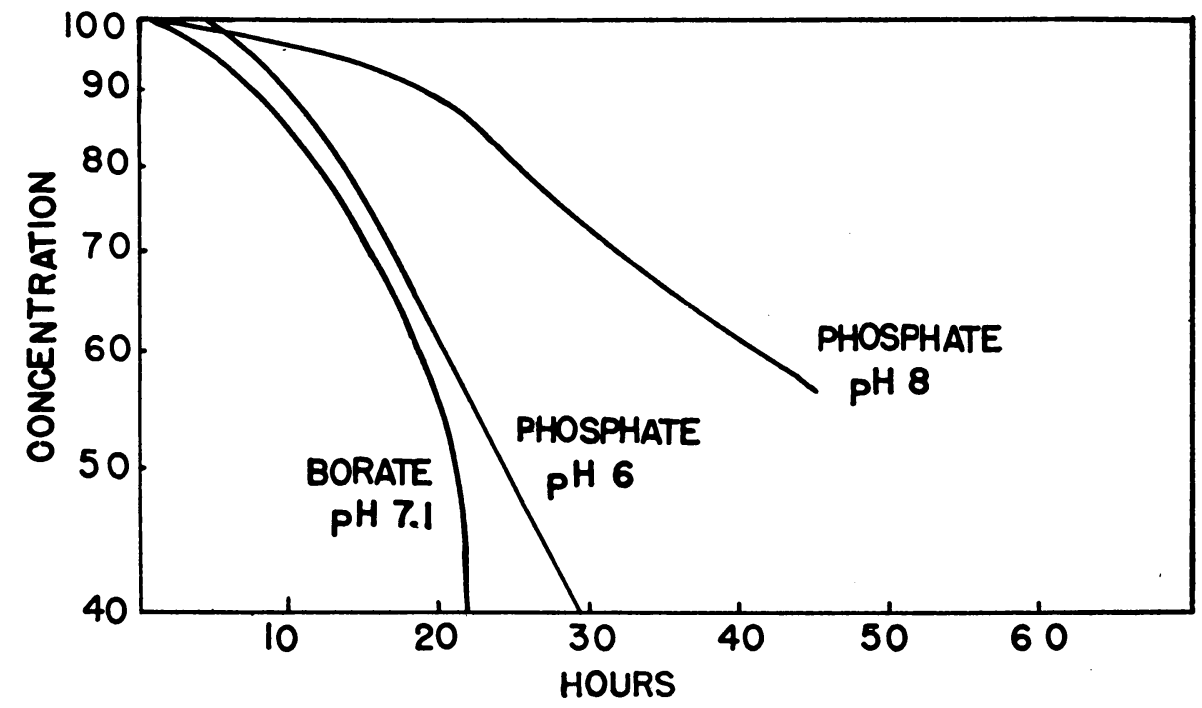

Fig. 6. Rate of carotene breakdown in cylinders of blanched carrot phloem, impregnated with standard phosphate buffers at $\mathrm{pH} 8$ and 6 and standard borate buffer at $\mathrm{pH}$ 7.1.

A neutral borate buffer did not stabilize the carotene. Blanched dice were soaked in the buffer for 20 minutes and kept moistened with it during incubation in moist air at $60^{\circ} \mathrm{C}$ (fig. 6, borate).

Acid and alkaline phosphate buffers differ in their protective properties. In blanched dice that were soaked in these buffers and moistened with them during incubation at $60^{\circ} \mathrm{C}$, the pigment was degraded more slowly than in unphosphated blanched dice. (Compare fig. 6 and the curves for summerharvested carrots of fig. 2.) Alkaline phosphate buffers protected the carotene much more than did the acid phosphate buffers. Neither acid nor alkaline phosphate buffers stabilized the pigment so well as the neutral phosphate buffer (fig. 5).

Buffer Treatments-Dehydrated Carrots. Results of phosphate treatmepts of dried carrots are at variance with each other. Winter-harvested carrots were diced and blanched. They were treated in vacuum for 5 minutes, with dilute solutions of $\mathrm{NaH}_{2} \mathrm{PO}_{4}$ and $\mathrm{Na}_{2} \mathrm{HPO}_{4}$ and standard buffer solutions at neutrality and at $\mathrm{pH} 8$. The dice were dried and stored in air at $40^{\circ} \mathrm{C}$ for 3 months. Carotene determinations were made monthly. Pigment degradation 
was faster in the phosphated dice than in the control blanched dice. After 3 months' storage, blanched dehydrated carrot dice still retained 83 per cent of their original carotene, whereas phosphated dice contained only 74 per cent. $^{5}$

Leaching of Soluble Phosphates. Leaching of soluble phosphate was tested under the experimental conditions (fig. 7). Phosphorus determinations were made according to the method described by Carolus (1938). In raw, untreated carrots the phosphate diffused very rapidly. About 10 per cent of

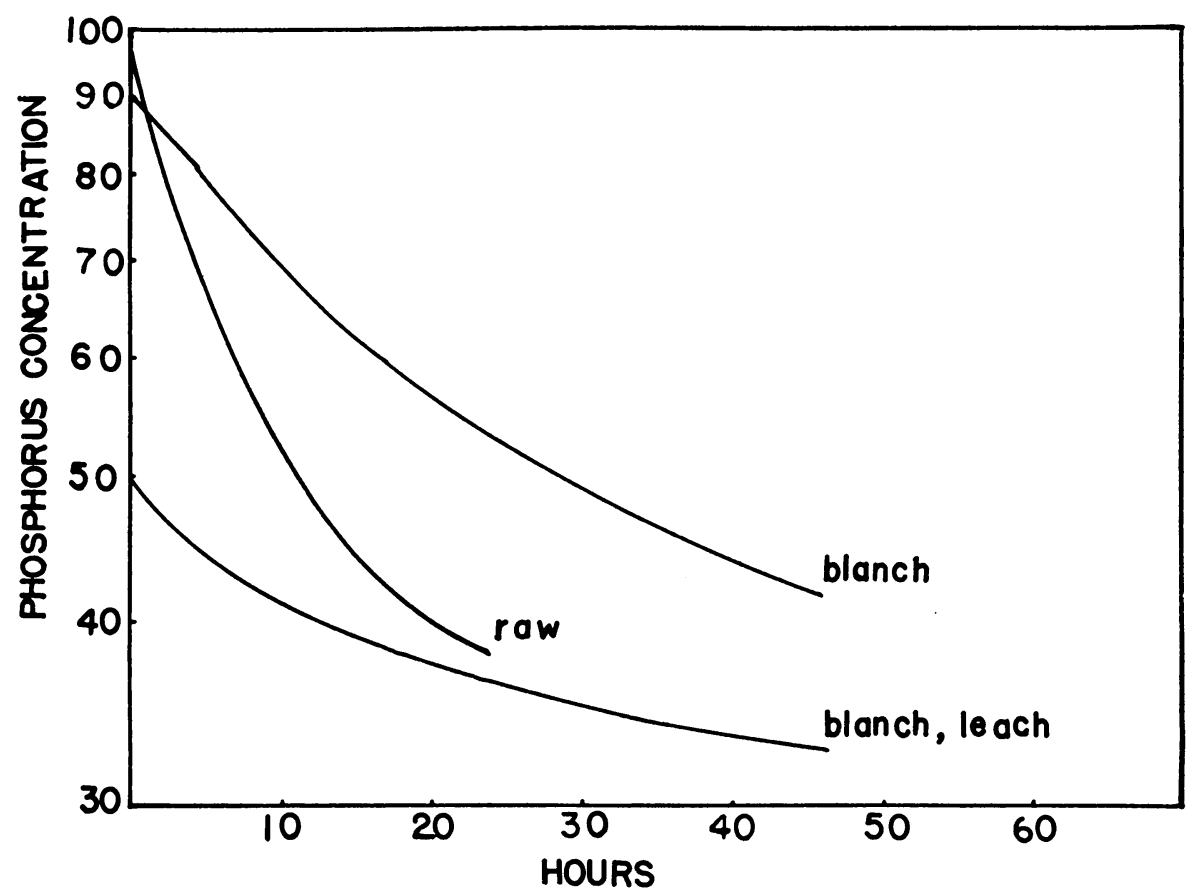

Fig. 7. Rate of diffusion of soluble phosphate from raw, blanched, and blanched leached cylinders of carrot phloem.

the soluble phosphorus was lost in blanching, and another 40 per cent in leaching. Phosphorus continued to diffuse from all carrot dice during incubation. Its rapid disappearance from untreated dice and its low value in the dice after washing could account for the greatly increased carotene breakdown in these carrots. (Compare fig. 6 with the previous figures.)

Blanched dice soaked in phosphate buffer contained at least three times the normal amount of phosphorus. Conceivably, phosphate may retard the breakdown of the carotene during the initial stages of the reaction. Phosphates and phosphoric acid are known to be antioxidants (Eckey, 1932).

Organic Solvents-Undried Carrots. Many antioxidants are fat-soluble, and in order to impregnate blanched carrot dice with them, a solvent must be used that will carry the antioxidant but will not remove the pigments. The

\footnotetext{
${ }^{5}$ These carrots were stored in an oven without forced draft. Localized convection currents over prolonged periods of incubation may account for a 100 per cent difference in paired samples, especially since the oven had to be crowded.
} 
stability of the pigments was tested after shaking the diced carrots in alcohols. These experiments were made with winter-harvested carrots, in which, because of the stability of the pigments, the effect of the alcohols on the inhibitor system could also be observed. The carotenoids are virtually insoluble in the concentrations of alcohols used; but small amounts of pigment were washed from the dice during the immersion in the alcohols.

Some blanched carrot dice were shaken for 30 minutes in 70 per cent ethyl alcohol, and others in 40 per cent methyl alcohol. All were then washed in several rinses of distilled water to remove as much alcohol as possible. During

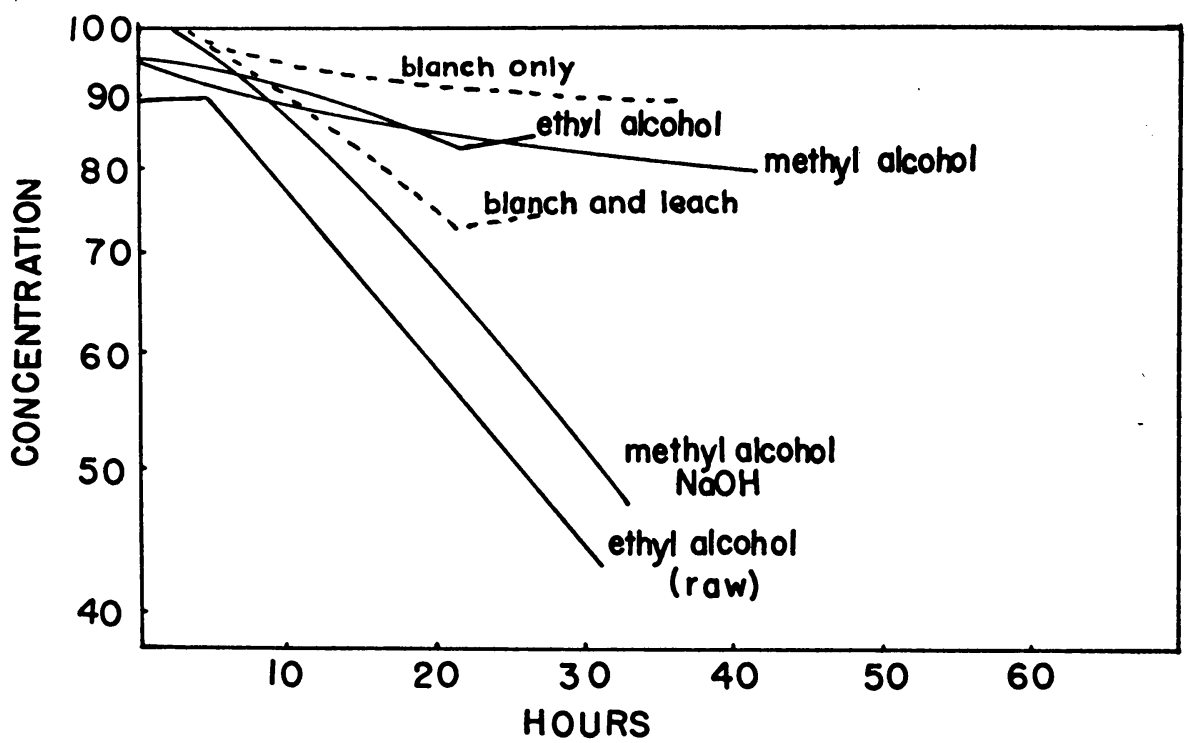

Fig. 8. Rate of carotene breakdown in blanched cylinders of winter-harvested carrots treated with alcohol after blanching. Dotted lines show the rate of carotene breakdown in blanched and blanched leached, winter-harvested carrots.

incubation they were moistened with distilled water. The breakdown of the pigment was comparable with the breakdown in dice that were washed in running water (fig. 8).

Raw dice infiltrated with 70 per cent ethyl alcohol were rinsed several times in distilled water and were moistened with it during incubation; see fig. 8, curve labeled "ethyl alcohol (raw)." A control experiment with untreated dice (curve not included in fig. 8), run concurrently, showed good agreement. The curves coincide at 4 and at 26 hours. The alcohol-infiltrated dice contained 10 per cent more pigment at 8 and 21 hours.

Blanched dice were infiltrated with 10 per cent $\mathrm{NaOH}$ in 40 per cent methyl alcohol. They were then washed, and were kept moistened with distilled water during incubation. The pigment decomposed very rapidly (fig. 8). Alkaline methyl alcohol must, in some way, destroy the inhibitor system that is present as a result of blanching.

Mineral Acids and Bases. The stability of the pigment in winter-harvested carrots facilitates a study of the influence of various substances on the in- 


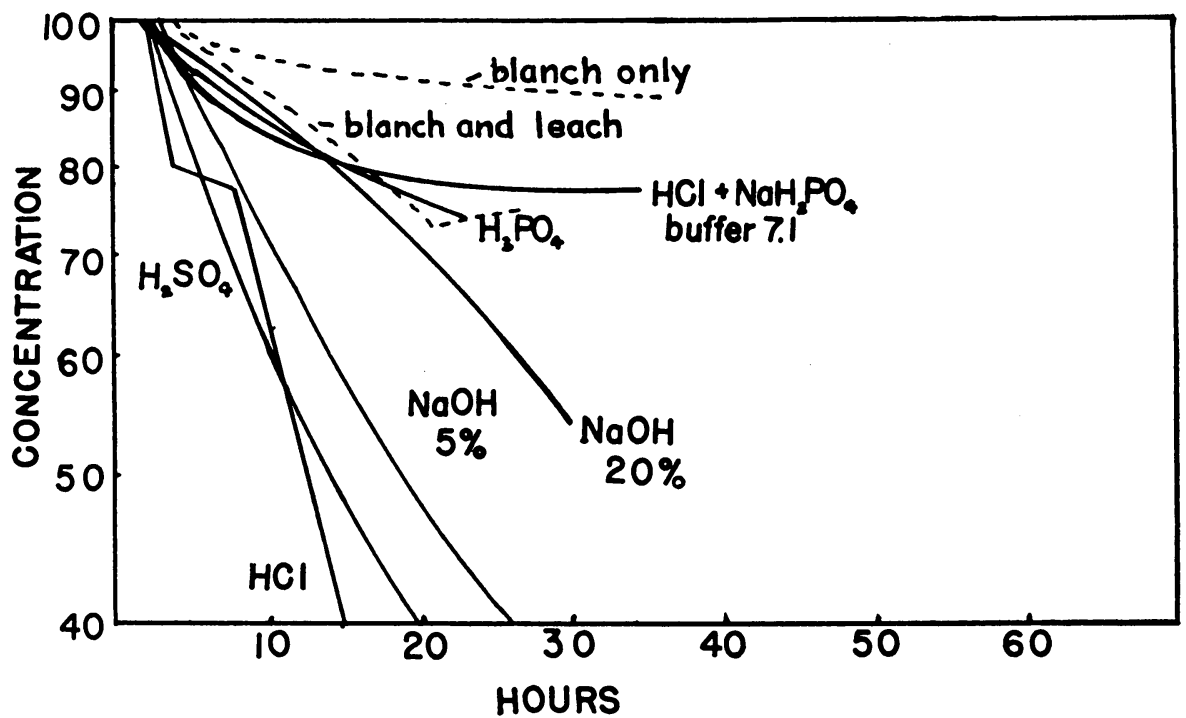

Fig. 9. Rate of carotene breakdown in blanched cylinders of winter-harvested carrots treated with inorganic acids after blanching. Dotted lines show the rate of carotene breakdown in blanched and blanched leached winter-harvested carrots.

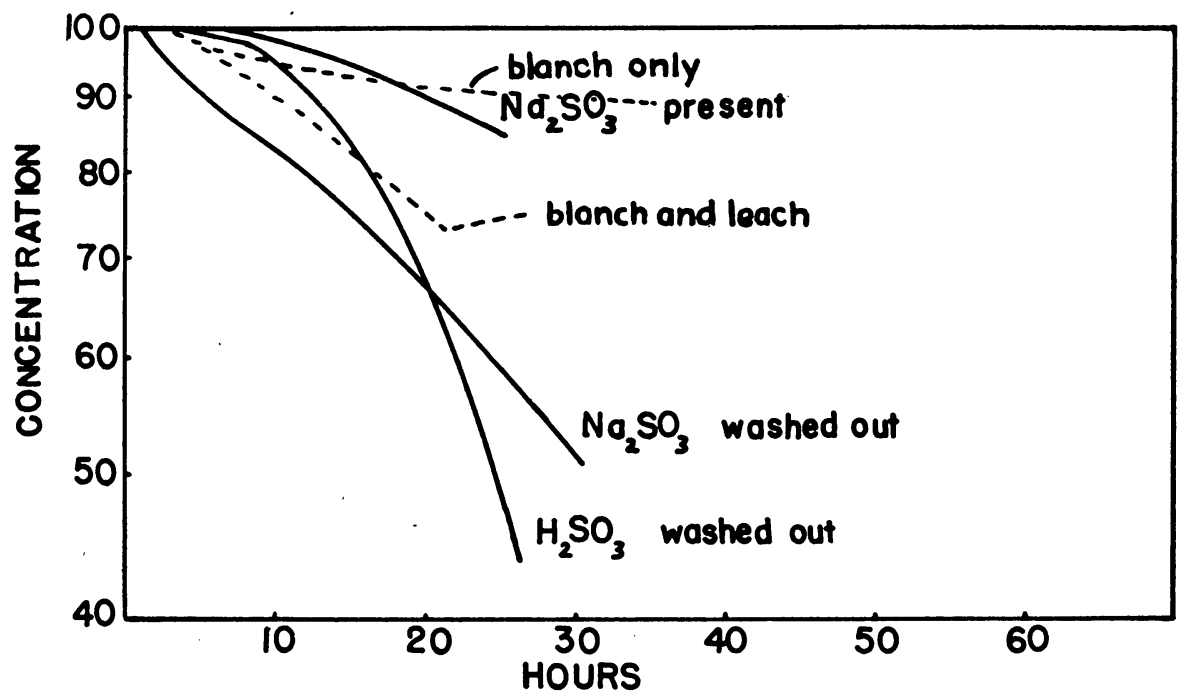

Fig. 10. Rate of carotene breakdown in blanched cylinders of winter-harvested carrots treated with $\mathrm{SO}_{2}$ after blanching. Dotted lines show the rate of carotene breakdown in blanched and blanched leached winter-harvested carrots.

hibitor system, which is released or activated by blanching. Blanched dice of winter-harvested carrots were soaked in aqueous solutions of the following materials : 5 and 20 per cent $\mathrm{NaOH}$; approximately 0.5 molar $\mathrm{H}_{2} \mathrm{SO}_{4} ; \mathrm{H}_{2} \mathrm{SO}_{3}$ to give approximately 1 part $\mathrm{SO}_{2}$ in 1,000 parts water; approximately 1 molar $\mathrm{HCl} ; 0.5$ per cent $\mathrm{Na}_{2} \mathrm{SO}_{3}$; and approximately 0.5 molar $\mathrm{H}_{3} \mathrm{PO}_{4}$. In all treatments except with one $\mathrm{Na}_{2} \mathrm{SO}_{3}$, the blanched dice were washed after soaking 
and were kept moist with distilled water throughout incubation. The results of these experiments are shown in figures 9 and 10. The presence of $\mathrm{Na}_{2} \mathrm{SO}_{3}$ during incubation protects the pigment (fig. 10, $\mathrm{Na}_{2} \mathrm{SO}_{3}$ present).

Pretreatment with $\mathrm{H}_{3} \mathrm{PO}_{4}$ and 20 per cent $\mathrm{NaOH}$ apparently does not affect the inhibitor system. The rate of pigment degradation compared well with the rate of breakdown in blanched and washed carrots (fig. 9).

Pigment decomposition was accelerated by soaking blanched dice previous to incubation in $\mathrm{H}_{2} \mathrm{SO}_{4}, \mathrm{H}_{2} \mathrm{SO}_{3}, \mathrm{Na}_{2} \mathrm{SO}_{3}, \mathrm{HCl}$, and 5 per cent $\mathrm{NaOH}$, when these were not present during incubation (figs. 9 and 10). The action of the inhibitor system present in the dice after blanching was, in some manner, destroyed or hampered by these substances.

Dice blanched and treated with $\mathrm{HCl}$ were washed in distilled water and soaked in neutral buffer solution. They were moistened with buffer throughout incubation. Pigment degradation was comparable with that of blanched and washed winter-harvested carrots (fig. 9, $\mathrm{HCl}+\mathrm{NaH}_{2} \mathrm{PO}_{4}$ buffer 7.1).

Organic Acids-Undried Carrots. Blanched dice of winter-harvested carrots were shaken for 30 minutes in solutions of various organic acids (Olcott and Mattill, 1936b). They were then drained and separated into two groups. One group (curves numbered 1 in figs. 10 and 11) was moistened with the acid during incubation. The second group (curves numbered 2 in figs. 11 and 12) was rinsed in distilled water after treatment with the acid and was moistened with distilled water during incubation. This procedure allowed the acid to diffuse from the dice, so that it was possible to differentiate between an interference with the action of the inhibitor and a stabilization of the pigment by the acid. The following organic acids were used : 0.1 molar oxalic acid ; 0.25 molar maleic acid ; nordihydroguaiaretic acid, 1 part in 3,500 parts 6 per cent ethyl alcohol ; 0.1 per cent gallic acid ; 0.1 per cent ascorbic acid ; and sodium salts of oxalic and maleic acids adjusted to neutrality by the addition of $\mathrm{NaOH}$.

Treatment with the sodium salts of oxalic and maleic acids had no beneficial effect (fig. 11). The results agreed well with those obtained from experiments on blanched dice of winter-harvested carrots. Soaking and incubation with sodium maleate greatly accelerated the degradation of the pigment.

Treatment with nordihydroguaiaretic acid, before and during incubation, markedly decreased the decomposition of the pigment (NDGA 1, fig. 12). When the nordihydroguaiaretic acid was washed out before incubation, the pigment was degraded more slowly than in blanched and washed winterharvested carrots (NDGA 2, fig. 12). Treatment with oxalic acid likewise appeared to protect the carotene, the results being about the same whether it was applied during incubation or only before (oxalic, 1 and 2, fig. 12). Gallic acid and ascorbic acid, when present during incubation, also protected the pigments. Washing after treatment with gallic and ascorbic acids gave results that approached those of blanched and washed winter-harvested carrots. Maleic acid, when present during incubation, produced similar results. Breakdown was always more rapid in the dice that were washed after infiltration with the acid than in those that remained unwashed. When maleic acid was washed from the dice and the carrots were moistened with distilled water during incubation, the breakdown was very rapid. 


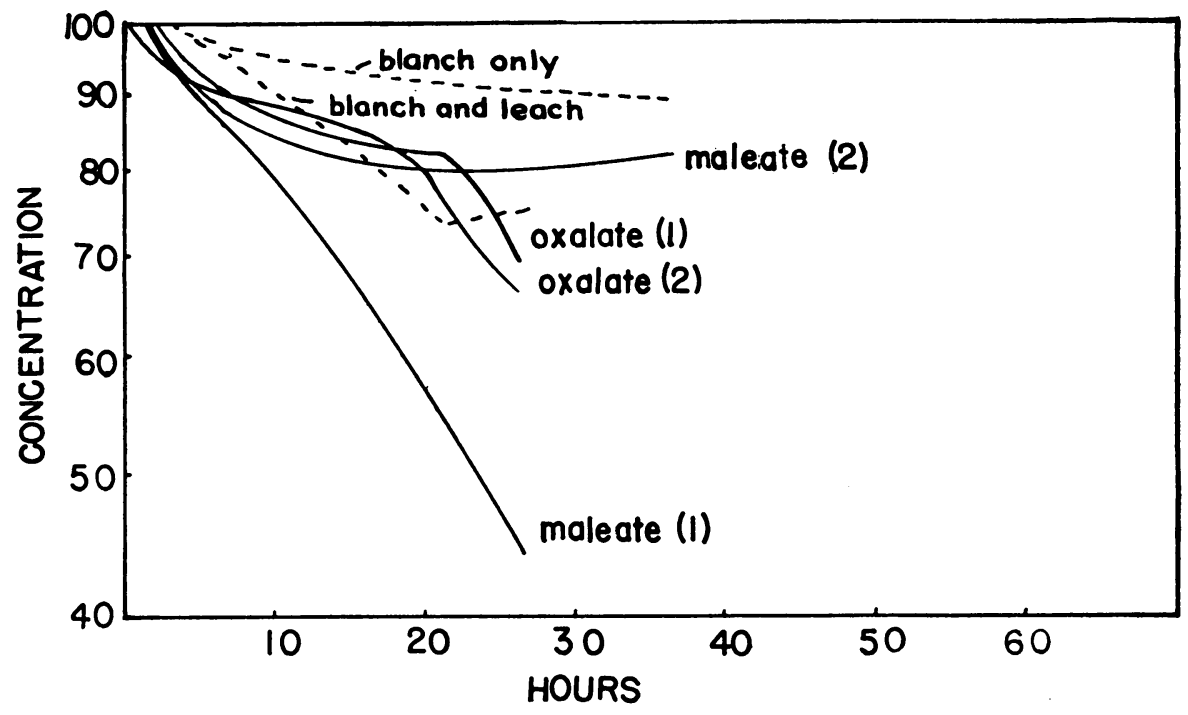

Fig. 11. Rate of carotene breakdown in blanched cylinders of winter-harvested earrots treated with sodium salts of maleic and oxalic acids after blanching and moistened with the salt (1) or with distilled water (2) during incubation. Dotted lines show the rate of carotene breakdown in blanched and blanched leached winter-harvested carrots.

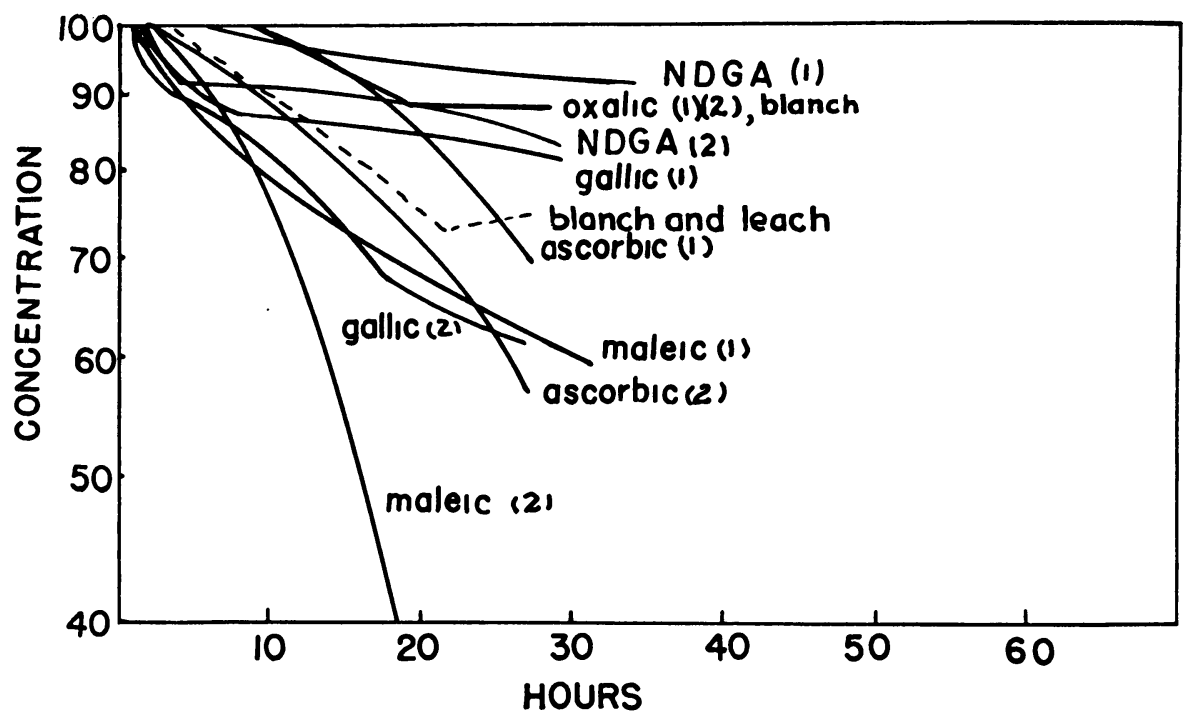

Fig. 12. Rate of carotene breakdown in blanched cylinders of winter-harvested carrots treated with dilute organic acids after blanching and moistened with the acid (1) or rinsed and moistened with distilled water (2) during incubation. Dotted line shows the rate of carotene breakdown in blanched leached winter-harvested carrots. The curve for the blanch experiments corresponds to the curve for the oxalic acid treatments. 
Organic Acids-Dehydrated Carrots. In another series of experiments, dice were soaked in these organic acids for 30 minutes. They were then dried to about 6 per cent moisture and stored at $40^{\circ} \mathrm{C}$ in air for 3 months. Carotene determinations were made monthly (fig. 13).

In dried carrots infiltrated with nordihydroguaiaretic or dipped in ascorbic acid after blanching, the pigment was very stable (NDGA and ascorbic dip, fig. 13). Soaking blanched carrot dice in ascorbic acid for 30 minutes under vacuum appeared to prolong the induction period; but breakdown of pigment

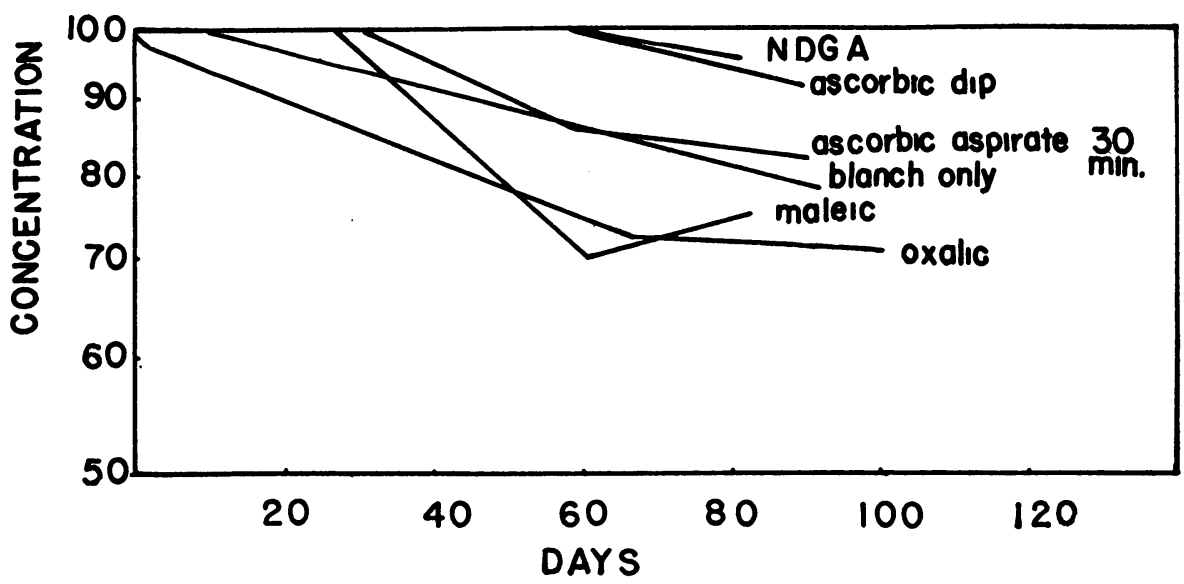

Fig. 13. Rate of carotene breakdown in diced, blanched, dried carrots treated with antioxidants after blanching and stored at $40^{\circ} \mathrm{C}$ for 3 months.

was rapid once it had started (ascorbic aspirate, $30 \mathrm{~min}$., fig. 13). Maleic acid also appeared to prolong the induction period. The pigment broke down rapidly after the treatment with oxalic acid (fig. 13).

\section{DISCUSSION}

Quality deterioration of dehydrated carrots appears to have at least two separate causes : deterioration in an oil-pigment fraction, and reactions in an aqueous fraction. Thus far, changes in the aqueous fraction have been considered only in a general way.

Results indicate that pigment breakdown may be stabilized by antioxidants. These results, however, are only preliminary; they need verification on other varieties of carrots grown in other localities. Spectrometric studies on pigment degradation might well revise the findings reported here. The investigations on rancidity changes in vegetable oils suggest other combinations of antioxidants that might improve the keeping qualities of dehydrated carrots. Some of these possibilities are being studied.

The present data indicate that antioxidants may be helpful in preserving the quality of dehydrated foods. Tressler and Du Bois (1944) and Bauernfeind and Siemers (1945) have shown that ascorbic acid, acting as an antioxidant, will prevent browning in peaches. According to Mills and Hart (1945), 0.9 per cent diphenylamine greatly retarded the breakdown of carotene in dehydrated oats and alfalfa. 


\section{SUMMARY}

The rate of pigment breakdown in raw carrots incubated in moist air at $60^{\circ} \mathrm{C}$ was uniform. It sometimes decreased after prolonged storage.

The rate of pigment breakdown in blanched carrots incubated in moist air at $60^{\circ} \mathrm{C}$ indicated that an inhibitor of some kind retarded the rate of breakdown during the initial stages of the reaction.

The pigments in carrot roots were more stable in winter-harvested than in summer-harvested carrots.

Neutral phosphate buffers, nordihydroguaiaretic acid, $\mathrm{Na}_{2} \mathrm{SO}_{3}$, oxalic acid, gallic acid, and ascorbic acid, when present in the blanched undried carrots during incubation, retarded the rate of degradation of the pigment.

Nordihydroguaiaretic acid and ascorbic acid retarded the rate of pigment degradation in blanched and dried carrot dice.

$\mathrm{HCl}, \mathrm{H}_{2} \mathrm{SO}_{4}, \mathrm{H}_{2} \mathrm{SO}_{3}$, and $\mathrm{Na}_{2} \mathrm{SO}_{3}$ destroyed the inhibitor system released by blanching. The pigment was degraded very rapidly when blanched dice were treated with these substances prior to incubation, when they were not present during incubation.

A substance that protects the carotene in carrot tissue after blanching may be leached from blanched dice by washing in running water.

\section{ACKNOWLEDGMENT}

Thanks are due to the Division of Truck Crops, to the members of the Division of Food-Technology, and to Mrs. Marjorie Morris who did much of the technical work in this experiment. 


\section{LITERATURE CITED}

BARNES, W. C.

1936. Effects of some environmental factors on growth and color of carrots. New York (Cornell) Agr. Exp. Sta. Memoir 186:1-36.

BAUERN FEIND, J. C., and G. F. SIEMERS.

1945. Adding ascorbic acid to peaches before freezing. Food Indus. 17:745-46.

Carolus, R. L.

1938. The use of rapid chemical plant nutrient tests in fertilizer-deficiency diagnoses and vegetable-crop research. Virginia Truck Exp. Sta. Bul. 98:1531-56.

ECKey, E. W.

1932. Composition of fatty matter and process of stabilizing same. U. S. Patent Office, Patent No. 1,993,152.

Koehn, C. J., and W. C. Sherman.

1940. The determination of vitamin A and earotene with the photoelectric colorimeter. Jour. Biol. Chem. 132:527-38.

Mills, R. C., and E. B. HART.

1945. Studies on the stabilization of carotene in dehydrated feeds and foods. Jour. Dairy Sci. 28:1-13.

Olcott, H. S., and H. A. Matrill.

1936a. Antioxidants and the autoxidation of fats. VI. Inhibitols. Amer. Chem. Soc. Jour. 58:1627-30.

1936b. Antioxidants and the autoxidation of fats. VII. Preliminary classification of inhibitors. Amer. Chem. Soc. Jour. 58:2204-8.

Strain, H. H.

1941. Unsaturated fat oxidase: specificity, occurrence, and induced oxidations. Amer. Chem. Soc. Jour. 63:3542.

SUMNER, R. J.

1942. Lipid oxidase studies. III. The relation between carotene oxidation and the enzymic peroxidation of unsaturated fats. Jour. Biol. Chem. 146:215-18.

Tressler, D. K., and C. Du Bois.

1944. No browning of cut fruit when treated by new process. Food Indus. 16:701, 763-65.

WEIER, T. E.

1944a. Carotene degradation in dehydrated carrots. I. Cytological changes in carotene and fat droplets under conditions favorable for carotene degradation. Amer. Jour. Bot. 31:342-46.

1944b. Carotene degradation in dehydrated carrots. II. Stability of carotene in carrot tissue kept in moist air at $60^{\circ} \mathrm{C}$. Amer. Jour. Bot. 31:537-44. 
